\title{
Cluster-induced crystallization of nano-silicon particles
}

\author{
J. Dutta, R. Houriet and H. Hofmann \\ Laboratoire de Technologie des Poudres \\ Département des Matériaux \\ École Polytechnique Fédérale de Lausanne \\ 1015 Lausanne, SWITZERLAND \\ H. Hofmeister \\ Max-Planck Institute of Microstructure Physics \\ Weinberg 2, D- 06120 Halle, GERMANY
}

\begin{abstract}
The possibility of producing from $20 \mathrm{~nm}$ to $100 \mathrm{~nm}$ sized silicon powders by plasma-induced reactions of silane including the formation of high-mass hydrogenated silicon anion clusters has been recently demonstrated. Careful HREM imaging showed circular contrast features, 1.5 to $2.5 \mathrm{~nm}$ in size, embedded in the amorphous particles which was attributed to the presence of medium range order in these regions. This nonhomogeneous atomic distribution with partially ordered regions of a couple of nanometer dimension became more pronounced upon annealing upto the onset of crystallization. This observation and the formation of fivefold crystallites thereafter indicate that the clusters serve as seeds in the amorphous-to-crystalline transition. (1997 Acta Metallurgica Inc.
\end{abstract}

\section{INTRODUCTION}

Nano-sized silicon powders are being increasingly studied in recent times for application in high performance ceramics and optoelectronics (1). Interesting improvement in the mechanical properties have been obtained for silicon based ceramics like silicon nitride, silicon carbide and silicon carbo-nitrides making use of nano-sized particles (2). Several techniques have been utilised for the synthesis of nano-sized powders but it is generally agreed that gas-phase condensation techniques lead to high purity materials. We have demonstrated the possibility of producing uniformly sized small silicon particles by plasmainduced dissociation of silane. Extensive work has been carried out in our laboratory and elsewhere to study the formation of these powders in the silane plasma (3). The growth mechanism of these powders are fairly well understood and now attempts are being made to understand the microstructure of the synthesized powders with respect to the variation in the plasma parameters (4). The silicon powders are formed by agglomeration of anionic silicon hydrides (5). In this report we will briefly elaborate on our earlier observation from Raman spectroscopy about the presence of clusters in the particles by the observation of these clusters by high resolution transmission electron microscopy and briefly discuss how the clusters act as seeds for crystallization (6). Attempt is being made to find the structure of these clusters and some of the structural mociels presently considered will be briefly discussed. 


\section{RESULTS \& DISCUSSION}

Details of the synthesis and characterization of the powders are available elsewhere $(1$, $3,5)$. The powders for this study were annealed in forming gas to avoid excessive oxidation. In-situ ion-mass spectroscopy suggested the formation of silicon powders in a PECVD process by the agglomeration of anions. Typically a few tens of anions form each cluster which is about $2-3 \mathrm{~nm}$ in size that ultimately lead to the formation of the primary particles (20-100 nm) in the powders synthesized. Laser light-scattering experiments shows that another clustering phase occurs when these ions achieve a size of about $2-3 \mathrm{~nm}$. Earlier, very narrow phonon modes were observed as shoulder of the characteristic Raman spectra of amorphous silicon in nano-sized silicon powders which were attributed to arise from localised modes (6). Selected area electron diffraction (SAED) during transmission electron microscopy showed halos typical of amorphous materials while, X-ray diffraction (XRD) patterns also showed amorphous features. Careful HREM imaging however points to the presence of circular contrast features of $1.5-2.5 \mathrm{~nm}$ in size embedded in an amorphous matrix as represented in figure 1 .

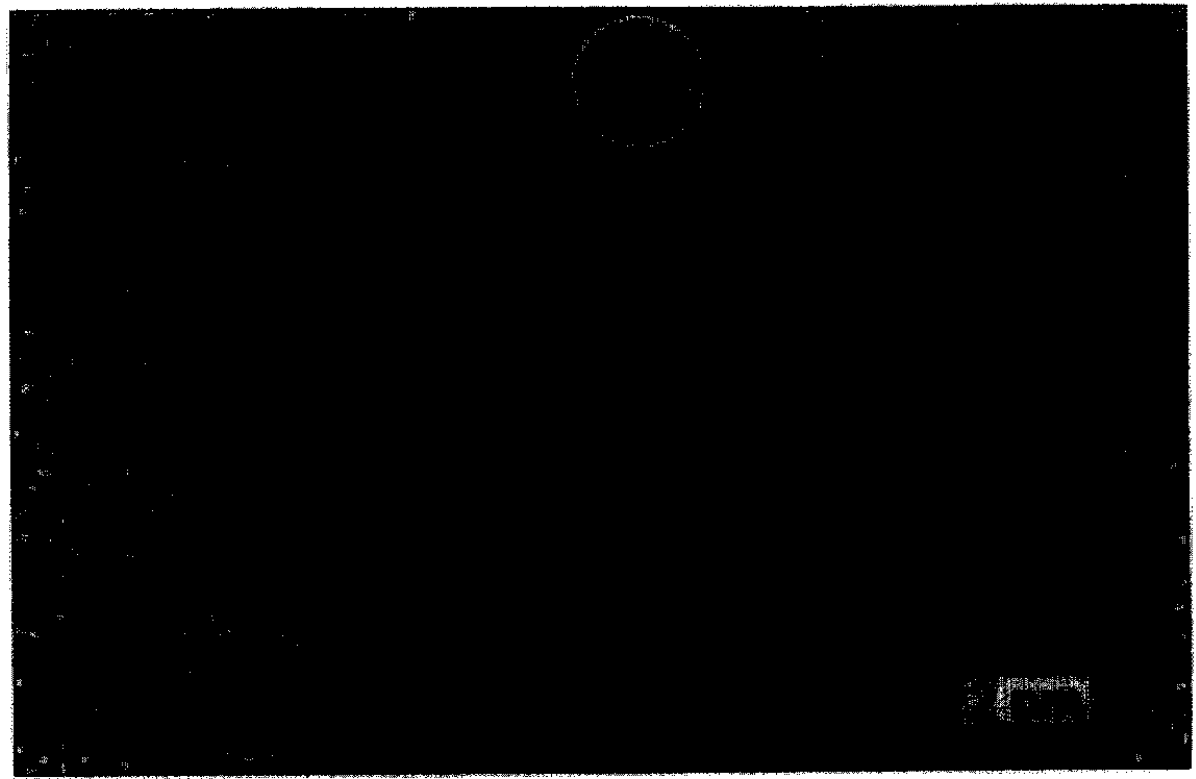

Figure 1. HREM image of a part of an amorphous Si particle which exhibits ring-like (marked by circles) and fringe-like (marked by arrows) contrast features, respectively.

Upon prolonged annealing at $600{ }^{\circ} \mathrm{C}$ for a few hours, the medium range ordered contrast features become more pronounced as shown in the dark field image in figure 2 , suggesting the possibility of these to have been the building block of the particles during synthesis. We observe bright specks arising from non-uniform atomic distribution, which agree very well with the circular contrast features as observed in the HREM images as shown in figure 1 . We can conclude that the clusters of $2-3 \mathrm{~nm}$ size, as observed from in-situ lightscattering experiments agglomerate to form the primary particles of the powder. 
The image contrast features obtained by HREM were attempted to replicate using a model with $100 \mathrm{Si}$ atoms, and the result is shown in figure 3 . The model is based on a 20 atoms core (having the shape of a pentagon dodecahedron), the 12 pentagonal faces of which are decorated by truncated pentagonal bipyramids (TPB). The tetrahedral bond is preserved with bond angles and bond lengths only slightly different from that of diamond cubic (dc) Si. Each of the 60 outer atoms have one unsaturated (dangling) bond. Always three neighboured TPB's surround a point at which a tetrahedron with dc lattice readily may nucleate. This cluster model first has been proposed by Gerstengarbe as the building block of a possible quasicrystalline phase of Si (7). The calculated contrast features agree reasonably well with the HREM observations and will be useful to understand the crystallization process in silicon nano-powder in further detail.

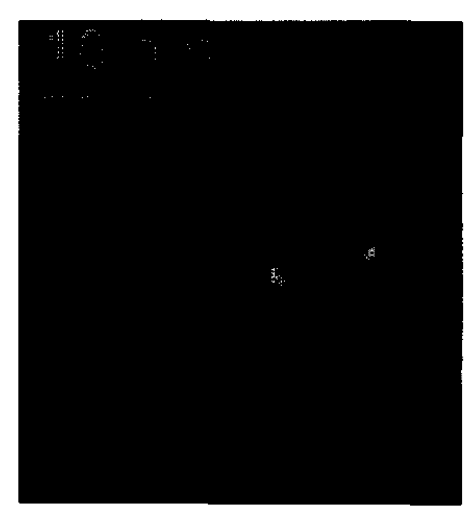

Figure 2: Dark-field image recorded by using the first diffusive ring of the diffraction pattern after annealing at $600^{\circ} \mathrm{C}$.

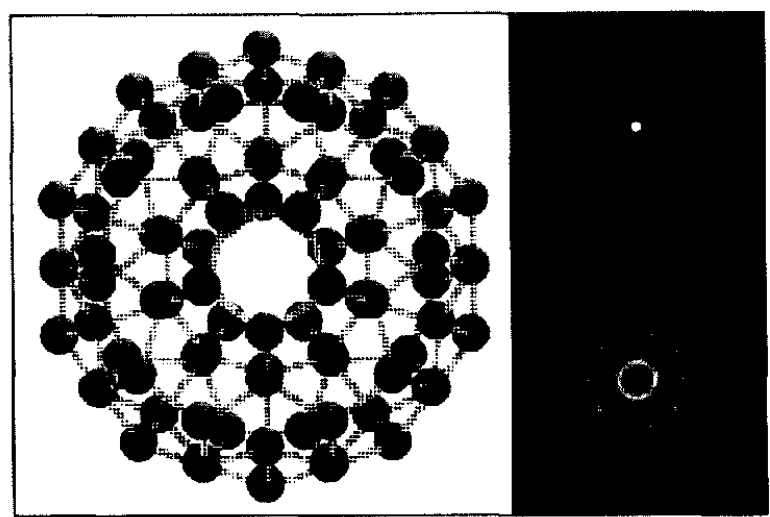

Figure 3: $\quad 100 \mathrm{Si}$ atoms cluster model and corresponding calculated image contrasts for two characteristic defocus settings.

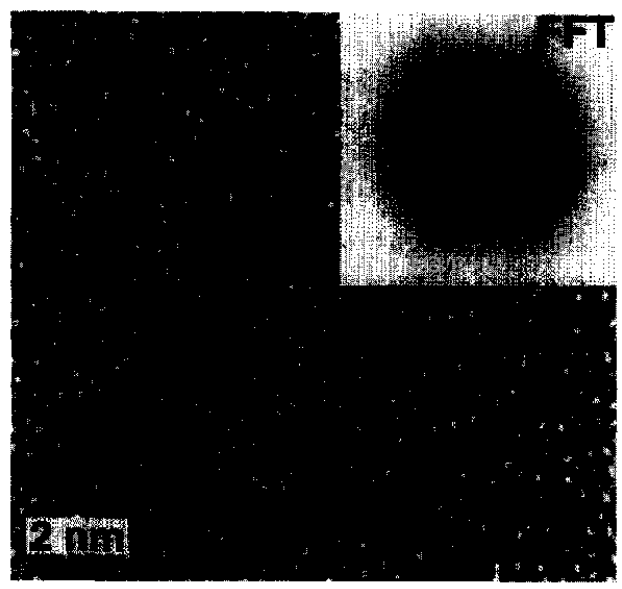

Figure 4. HREM image of a typical crystallite at the onset of crystallization; inset: FFT of the crystalline region 
In figure 4 we have shown a typical crystallite at the onset of crystallization. Fast Fourier transform (FFT) of the image clearly exhibits faint [111] spots. It is quite apparent from the size of the crystallite that the clusters act as nucleation centers for the crystallization process. The crystallization is confined to the primary particle and considerable fraction of the sample does not transform into the crystalline form. It is suggested that the clusters which form the primary particles act as the seed for the crystallization process leading frequently to five-fold twinned crystallites. After annealing at temperatures upto $\sim 900^{\circ} \mathrm{C}$ crystallization proceeds mainly by growth twinning resulting in a heavily faulted structure of the particles.

\section{CONCLUSIONS}

We have shown that clusters which form the building block of silicon nano-particles govern the crystallization phenomena. These results are of interest to both the structural and functional ceramic applications as well as for the study of confinement effects in silicon nano-crystals.

\section{ACKNOWLEDGEMENTS}

This work has been partially funded by the Fonds National Suisse under contract no. $2100-039361.93 / 1$. Thanks are due to $\mathrm{D}$. Timpel for providing the atomic coordinates of the cluster model.

\section{REFERENCES}

1. H. Hofmeister, J. Dutta and H. Hofmann, Phys. Rev, B,54 (1996) 2856.

2. e.g. "Silicon Nitride'93", eds. M. J. Hofmann, P. F. Becher and G. Petzow, Trans Tech Publications, Switzerland (1994).

3. J. Dutta, R. Houriet, H. Hofmann, J. -L. Dorier, A. A. Howling and Ch. Hollenstein, Proc. of 6th European Conference on Applications of Surface \& Interface Analysis (ECASIA 95), John Wiley \& Sons, Chichester and New York, eds. H. J. Mathieu, B. Reihl and D. Briggs (1996), pg. 483-486.

4. Ch. Hollenstein, J.-L. Dorier, J. Dutta, L. Sansonnens and A. A.Howling, Plasma Sources Sci. Technol. 3 (1994), 278-285.

5. J. Dutta, H. Hofmann, R. Houriet, H. Hofmeister and Ch. Hollenstein, Colloids \&

Surfaces (1996) in press.

6. J. Dutta, W. Bacsa and Ch. Hollenstein, J.Appl.Phys. 77 (1995) 3729-3733.

7. C. Gerstengarbe, Publications of the 12. Electron Microscopy Conference, Dresden, 1988, pg. 481-482, A174. 